\title{
Logistische Materialvorhaltung der Polytraumaversorgung im Schockraum aus unfallchirurgischer Sicht unter Berücksichtigung des Status der Klinik: Überregionales Traumazentrum AKH Celle (Schwerpunktversorger)
}

\author{
Abd Elgani Haj Yahya, Ulf Culemann
}

\section{Einleitung}

Die Behandlung des schwerverletzten Patienten in einem überregionalen Traumazentrum ist ein komplexes Zusammenspiel aus den vorhandenen Ressourcen (Personal, Material, Aus- und Fortbildung [1 -4]) und der Zusammenarbeit des Behandlungsteams bei Eintreten der Behandlungssituation. Dieses Zusammenspiel kann aber nur bei einer exakt geplanten logistischen Materialvorhaltung in Schockraum und Notfall-OP erfolgreich funktionieren. Neben einem gut ausgebildeten Schockraumbehandlungsteam bestehend aus Unfallchirurgen, Anästhesieteam und Radiologen (Arzt und Pflege) und einem mit den Arbeitsabläufen vertrauten Pflegepersonal sowie Funktionsassistenten (Radiologie, Labor) in der Zentralen Notaufnahme (ZNA) ist auch eine schnell und leicht erreichbare Materialnutzung und Materialorganisation wichtig. Daher hat heutzutage insbesondere die notwendige Lagerkapazität, die Erreichbarkeit und Wiederverfügbarkeit der Instrumente und Verbrauchsmaterialien in kürzester Zeit eine ebenso hohe Bedeutung für die zielgerichtete und zeitsparende Versorgung eines Polytraumas wie die Aus- und Weiterbeildung der beteiligten Mitarbeiter. Letztlich beeinflusst somit auch eine gute Materialvorhaltung indirekt die Prognose des behandelten Patienten.

Die notwendige Ausstattung der Klinik mit Gerätschaften und die Einhaltung der hierfür nötigen Material- und Lagerhaltung in Schockraum und OP sind einerseits durch das Weißbuch Schwerverletztenversorgung [5,6] festgelegt und andererseits durch Vorgaben der Deutschen Gesetzlichen Unfallversicherung (DGUV) zwingend einzuhalten [7], um einen bestimmten Status der Versorgungsstufe zu erreichen und zu halten (Lokales, Regionales bzw. Überregionales Traumazentrum nach DGU-Einteilung, Zulassung zum Durchgangsarztverfahren [DAV], Verletzungsartenverfahren [VAV] bzw. Schwerstverletzungsartenverfahren [SAV] der DGUV) [5-7]. In regelmäßigen 3-Jahres-Zyklen werden diese Stufen durch interne und externe Prüfer auditiert und bestätigt bzw. korrigiert. Die zielgerichtete Funktion der Arbeitsabläufe in der Polytraumbehandlung wird dabei ebenso begutachtet wie die ausreichende Personalausstattung der Klinik und die korrekte Vorhaltung und Verfügbarkeit der notwendigen Materialien, die für eine erfolgreiche Behandlung benötigt werden.
Wie diese Gerät- und Materialausstattung bereitgestellt und wie die Nachhaltigkeit der Gebrauchsfähigkeit durch die jeweilige Klinik sichergestellt wird, um den Auditierungen und Vorgaben standzuhalten, ist nicht festgelegt und bedarf daher guter und auf die jeweilige Klinik abgestimmter Behandlungsabläufe ebenso wie der hierfür notwendigen Materialvorhaltung. Alle Mitarbeiter sämtlicher Fachrichtungen in der Notaufnahme müssen dabei regelmäßig in die Materialnutzung und -lagerung eingewiesen werden und ebenso muss die Nachbestellungslogistik regelmäßig geprüft und angepasst werden.

Ziel des vorliegenden Beitrags ist es daher, die notwendige Materialvorhaltung in Schockraum und OP für die primäre Versorgung eines schwerverletzten Patienten aus Sicht eines auditierten Überregionalen Traumazentrums der DGU zu beschreiben und die notwendigen Prozessschritte für eine zielgerichtete Primärversorgung des schwerverletzten Patienten darzustellen. Am Beispiel des Überregionalen Traumazentrums am AKH Celle wird dargestellt, wie aktuell Materialwirtschaft und Logistik umgesetzt werden, um eine optimale (Material-)Versorgung für Schwerverletzte zu gewährleisten.

\section{Schockraumversorgungsablauf}

Der Ablauf der Versorgung der Polytraumata beginnt schon bei der Polytraumaanmeldung [2]. Die Anmeldung erfolgt durch die Rettungsleitstelle über den 1. Dienstarzt der Unfallchirurgie oder die ZNA-Pflege über eine zentrale Rufnummer. Diese Anmeldung löst eine darauf aufbauende Kaskade aus ( $\boldsymbol{A}$ Abb. 1). Diese Kaskade ist ein gemeinsam festgelegter und letztlich selbstständig ablaufender Prozess, der dennoch regelmäßig geschult wird, um auch neue Teammitglieder anzuleiten. Jeder Mitarbeiter des Traumazentrums ist über den Ablauf informiert und geschult. Dies gilt insbesondere auch für die Alarmierungskette.

Um die Qualität dieses Ablaufes jederzeit zu gewährleisten, werden

- mehrmals im Jahr Schockraumtrainings zur Übung durchgeführt und hier insbesondere neue Mitarbeiter gezielt geschult. Hierzu wird jeweils eine strukturierte Nachbesprechung mit allen Mitarbeitern geführt. Die Übung wird mit Videoaufzeichnung begleitet und im Rahmen einer Schockraumzirkel-Fortbildung präsen- 


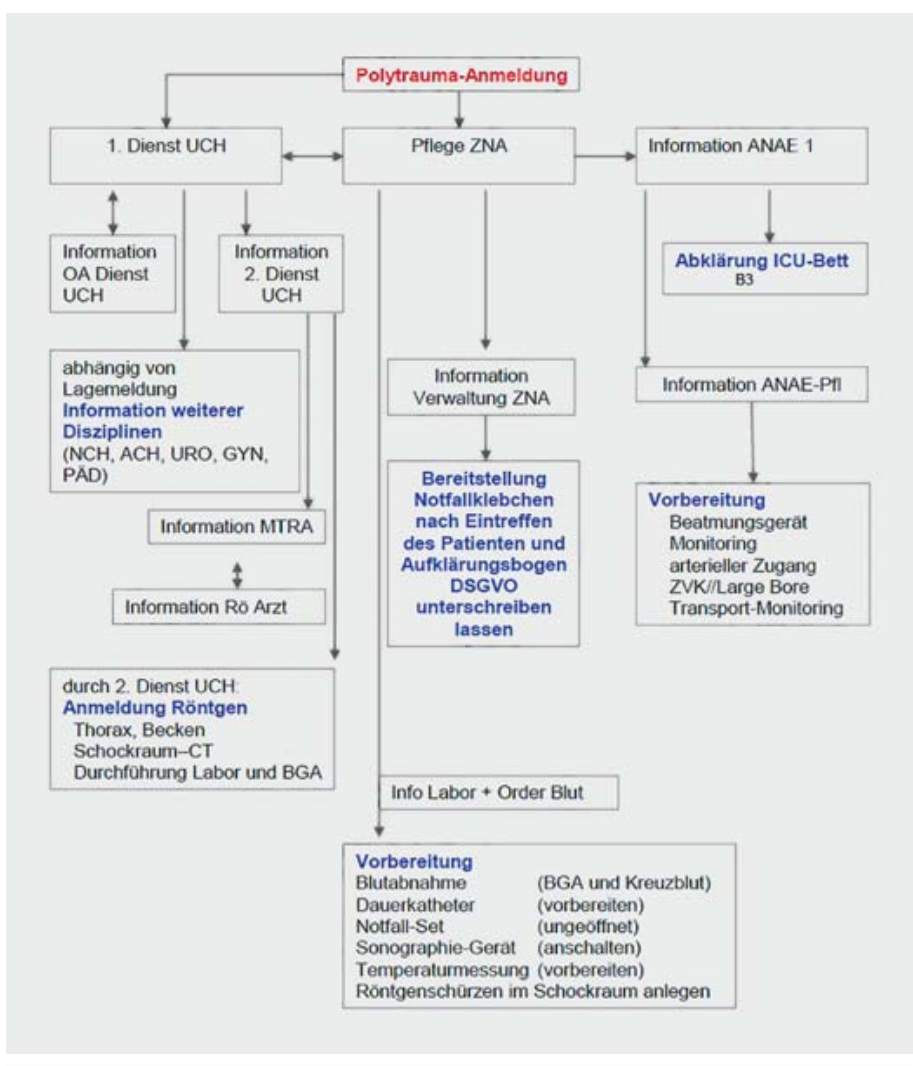

- Abb. 1 Schema: Polytrauma: vorbereitende Maßnahmen am AKH Celle. tiert und gemeinsam diskutiert ( $\bullet$ Abb. 2). Durch interne Audits wird die Umsetzung durch das Qualitätsmanagement begleitet.

- Schockraumzirkel mindestens 2x/Jahr intern und extern gemeinsam mit Rettungsleitstelle und Rettungsdienst zur weiteren Optimierung der Abläufe bei Schockraumanmeldung und Behandlung im Schockraum durchgeführt.

- klinikinterne M \& M-Konferenzen (M \& M: Morbidity \& Mortality) unter Einbeziehung der Mitarbeiter der unfallchirurgisch-orthopädischen Klinik und der Mitarbeiter des Traumazentrums (ZNA, Anästhesie, Radiologie, Intensivstation) abgehalten.

- Mitarbeiter zu den ATLS-Provider-Kursen (ATLS ${ }^{\circledR}$ : Advanced Trauma Life Support) bei Eintritt in die Klinik geschickt und ATLS-Refresher-Kurse genutzt, um die Fortbildung im ärztlichen Bereich auch langfristig zu sichern [6-8].

- durch die Teilnahme an der Notfallkonferenz der DGU und Teilnahme am TDSC-Kurs ${ }^{\circledR}$ (TDSC: Terror and Desaster Surgical Care) in Ulm einzelne Mitarbeiter der Klinik weitergebildet

Die Anmeldekriterien für eine Polytraumaanmeldung wurden im Schockraumzirkel gemeinsam mit der Rettungsleitstelle und dem Rettungsdienst in einem sog. Schockraumanmeldebogen festgelegt. Durch die Differenzierung zur Schockraumanmeldung von Patienten, die zwar den Mechanismus nach der S3-Leitlinie $[9,10]$ erfüllen, aber kein A-E-Problem nach ATLS haben [8], konnte auch die zeit- und personalaufwendige Schockraumteamalarmierung auf das notwendige Maß reduziert werden. Grundsätzlich kann natürlich aus einer
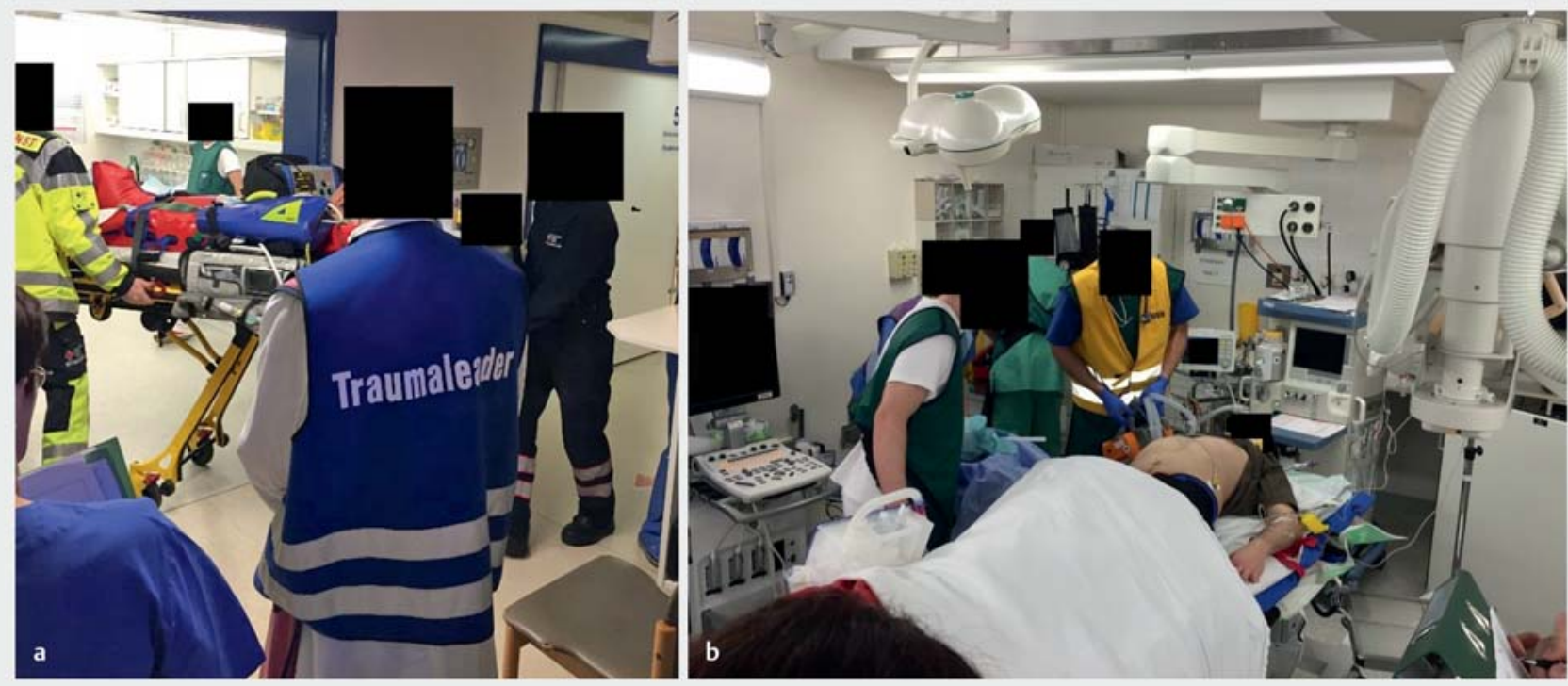

- Abb. 2 a Schockraumtraining mit Übungspatient + Rettungsdienst. Beachte Traumaleader-Weste zur eindeutigen Erkennung! b Schockraumtraining am Übungspatienten. 

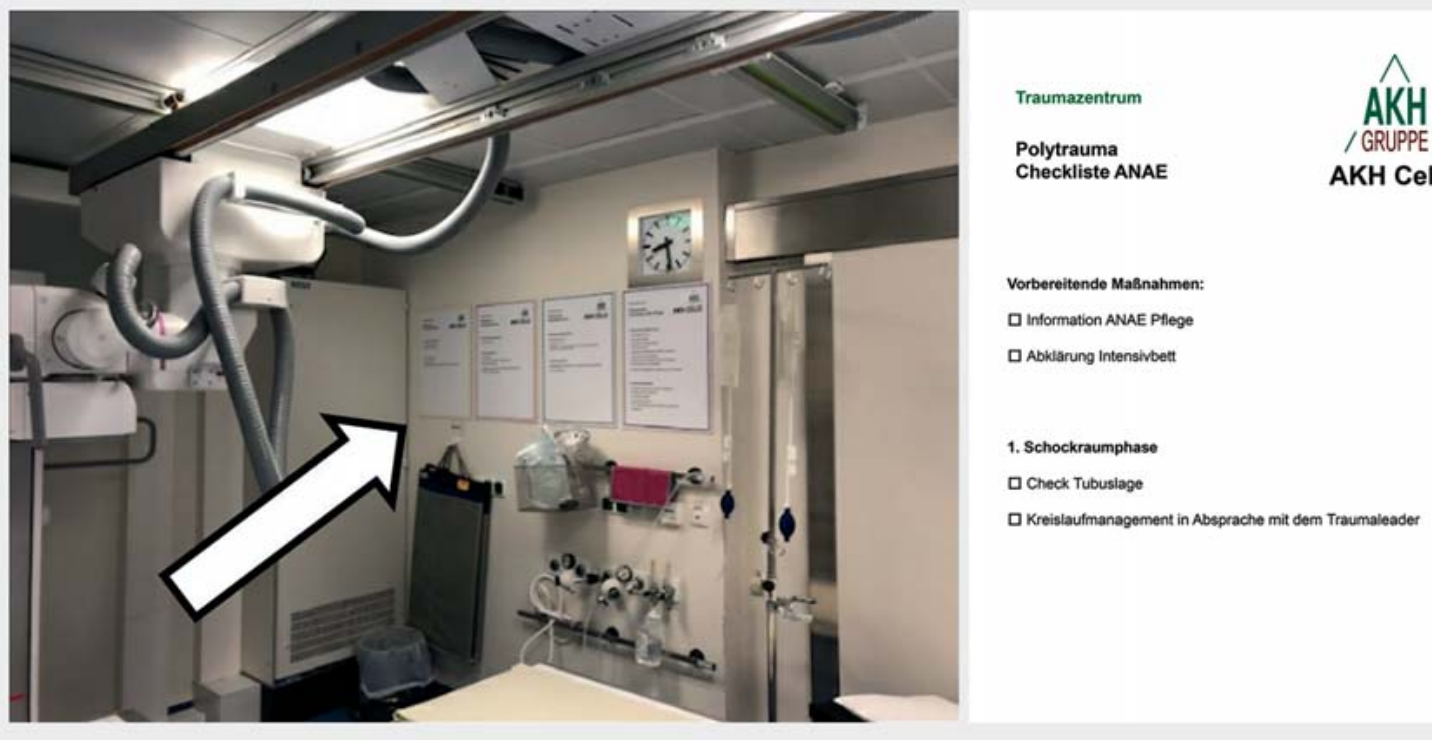

- Abb. 3 Schilder im Schockraum mit Abbildung der vorgesehenen Aufgabenverteilung; rechtsseitige Vergrößerung z. B. Checkliste ANAE = Checkliste für die Mitarbeiter der Anästhesie.

„Schockraumanmeldung“ jederzeit eine „Polytraumaversorgung“ entstehen, aber nach dem „Alles-oder-Nichts“Prinzip wird bei einer Polytraumaanmeldung grundsätzlich das komplette Schockraumteam zusammengerufen, bis der Traumaleader zumeist gemeinsam mit dem Arzt für Anästhesie die Polytraumabehandlung für beendet erklärt und das Team aus der Polytraumabehandlung wieder entlässt.

Die einzelnen vorgelagerten Aufgaben der Mitarbeiter vor und während der Schockraumversorgung wurden neu definiert und durch Anfertigung großer Schilder im Schockraum zur Darstellung gebracht ( $\triangleright$ Abb. 3 ). Die Aufgaben beziehen sich sowohl auf die vorbereitenden Maßnahmen als auch auf die Aufgaben nach Eintreffen des Patienten. Die Standardisierung der Schockraumversorgung basierend auf dem Konzept des ATLS [8] war hierfür eine unabdingbare Voraussetzung zur Verbesserung der Versorgungsqualität schwerverletzter Patienten unter Verkürzung der notwendigen Behandlungszeit im Schockraum. Hierzu wurde auch eine fundierte Weiterbildung im Kurssystem für alle Mitarbeiter angestrebt, damit alle im Schockraum tätigen Mitarbeiter „die gleiche Sprache“ sprechen. Ein regelmäßiges innerklinisches Training schwieriger Situationen im Schockraum wird unter Supervision eines erfahrenen Tutors mindestens 2mal im Jahr durchgeführt. Ziel dieser Maßnahmen, und damit auch ein messbarer Ergebnisparameter, soll dabei die Senkung der Letalität im Schockraum sein.
Das Basisteam Schockraum besteht aus:

- Oberarzt Unfallchirurgie (Zusatzweiterbildung Spezielle Unfallchirurgie)

- 1. Dienst Unfallchirurgie (Facharzt Orthopädie und Unfallchirurgie oder Facharzt Chirurgie bzw. Facharztstandard)

- 2. Dienst Unfallchirurgie (Weiterbildungsassistent/in)

- Dienst Anästhesie (Facharzt Anästhesie oder Facharztstandard)

- Anästhesiepflegekraft

- 2 Pflegekräfte der Zentralen Notaufnahme

- MTRA der Abt. für Radiologie

Nach Ankunft des Polytraumas folgt der Behandlungsablauf in der vorher festgelegten und trainierten nachfolgenden Reihenfolge:

1. Alarmierung

2. personelle Besetzung des Schockraums (siehe Basisteam Schockraum)

3. Übernahme des Patienten im Schockraum vom Notarzt/Rettungsteam

4. Phase 1 (Umlagerung, Basis-Evaluation ABCDE, 1.3. Minute)

5. Phase 2 (Sicherung Vitalfunktionen, Basisdiagnostik (Thorax, Becken), Basismonitoring, lebensrettende Notfallmaßnahmen, 3.-10. Minute)

6. initiale Labordiagnostik

7. Phase 3 (je nach Zustand des Patienten: Sofort-OP nach Damage-Control-Prinzip, Trauma-Scan-CT, ggf. erweiterte Diagnostik mit konventionellem Röntgen, ggf. Early-total-Care-OP) 


\section{Notwendige Gerätschaften und Mate- rialien für die Polytraumaversorgung}

Die logistische Materialvorhaltung basiert auf der Versorgung nach dem ATLS-Schema [8] gemäß „ABCDE“-Regel und der S3-Leitlinie „Schwerverletztenversorgung“ [1] basierend auf dem Weißbuch der Deutschen Gesellschaft für Orthopädie und Unfallchirurgie [5,6]. Das ABCDE ist eine Strategie zur Untersuchung und Versorgung kritisch kranker oder verletzter Patienten auf der Basis einer Prioritätenliste $[9,10]$. Es ist ein integraler Bestandteil internationaler Schulungskonzepte von NAEMT (National Association of Emergency Medical Technicians), AHA (American Heart Association) [11, 12] und ERC (European Resuscitation Council) [13].

Im Folgenden werden die einzelnen Aspekte des ABCDE mit ihren dazugehörigen Materialien aufgeführt:

Vorbereitende Maßnahmen und Bereitstellung von Gerätschaften im Schockraum bei Anmeldung:

- Beatmungsgerät

- Defibrillator

- Monitoring

- Ultraschallgerät

- EKG-Gerät

- Pulsoxymetrie

- Röntgengerät, Röntgenschürzen

- CT-Voranmeldung

- Traumaleader-Weste anlegen

- Krankenhausinformationssystem zur Patienteneingabe vorbereiten

- Patientenliege

- Besteck zur Entkleidung des Patienten

- Stethoskop

- Thermometer

- Taschenlampe

Notwendige Materialien, die im Schockraum verfügbar sein sollen:

- Thoraxdrainagen (am besten auf separatem Lagerungswagen)

- Perikardpunktionssieb

- Volumentherapie

- Druckverbandmaterial

- Beckengurt (Beckenzwinge)

- Dauerkatheterset

- Blutentnahmeset

- Tetanusimpfung (aktiv und passiv)

- Antibiose (Cephalosporin der 2. Generation)

- Splintschiene, Luftkammerschiene

- Handschuhe, Kittel (sterile Einmalsets)

- Patientenlaken, CT-Lagerungstuch, Wärmedecke

- Aufbewahrung für Patienteneigentum

- stichsicherer Abwurf
Bei Eintreffen des schwerverletzten Patienten wird direkt nach der Übergabe durch den Notarzt nach dem ABCDESchema des ATLS vorgegangen. Nachfolgend sind die einzelnen Abschnitte kurz erläutert und die dafür notwendigen Instrumente, Geräte und Sets werden dargestellt.

\section{A - Airway (Atemweg)}

Für die Beatmung sind nicht nur ein Stethoskop, ein Kapnometriegerät und eine Pulsoxymetrie erforderlich, sondern auch ein Set zur Anlage einer Thoraxdrainage (n. Bülau; • Abb. 4) mit zugehörigen Drainagen und Sogsystem und ggf. eine Nadelkompression zur Notfalldekompression eines Spannungspneumothoraxes (Monaldi-Drainage) sowie ein System für die Perikardpunktion und ein BGA-System zur Kontrolle der Beatmung und ersten Erfassung des Blutverlustes (Hb-Messung).

\section{B - Breathing (Beatmung)}

Zur Atemwegssicherung gehören die apparative Überwachung mit Transportmonitor, ein Beatmungsgerät, ein Endotrachealtubus bzw. eine Trachealkanüle, eine Larynxmaske, Druck- und Flowgeneratoren zur Atemsteuerung, eine Gesichtsmaske sowie ein Sauerstoffanschlussschlauch und $\mathrm{CO}_{2}$-Messgerät ( $\triangleright$ Abb. 5).

\section{C - Circulation}

Zur Stabilisierung des Kreislaufs gehören insbesondere eine Notfall-Beckenschlinge bzw. ein-Beckengurt. Diese Notfall-Tools werden zumeist bereits präklinisch am Notfallort angelegt und haben letztlich aufgrund ihrer einfachen Handhabung inzwischen die Notfall-Beckenzwingen aus dem Schockraum weitestgehend in den NotfallOP „verdrängt“ [14]. Die Anwendung der Beckenzwinge ist dabei indikationsspezifisch nicht direkt vergleichbar mit der Anwendung eines Beckengurtes. Vielmehr ergänzen sich diese Instrumente sinnvoll. Während der Beckengurt als Notfallmaßnahme bereits auf dem Transport für einen geringeren Volumenverlust beim Patienten und damit für eine verbesserte Kreislaufsituation sorgt [14] und somit dem Behandlungsteam mehr Zeit für erste Untersuchungen, Diagnostik und Maßnahmen verschafft (Pat. kommt einfach kreislaufstabiler im Schockraum an), kann die Beckenzwinge insbesondere für die mechanische Stabilisierung des hinteren Beckenrings im Notfall-OP eingesetzt werden und somit den fortgesetzten Blutverlust verhindern [15].

Weitere Materialien für die Aufrechterhaltung des Kreislaufs beim Patienten sind Druckverband und Tourniquet sowie Luftkammerschienen zur Extremitätenruhigstellung (für Erwachsene und für Kinder).

Zur Notfalldiagnostik auf freie Flüssigkeit im Abdomen oder eines Perikardergusses ist ein Sonografiegerät notwendig ( $\triangleright$ Abb. $\mathbf{6}$ ). Sets zur Anlage von arteriellen und zentralvenösen/periphervenösen Zugängen zur invasiven 


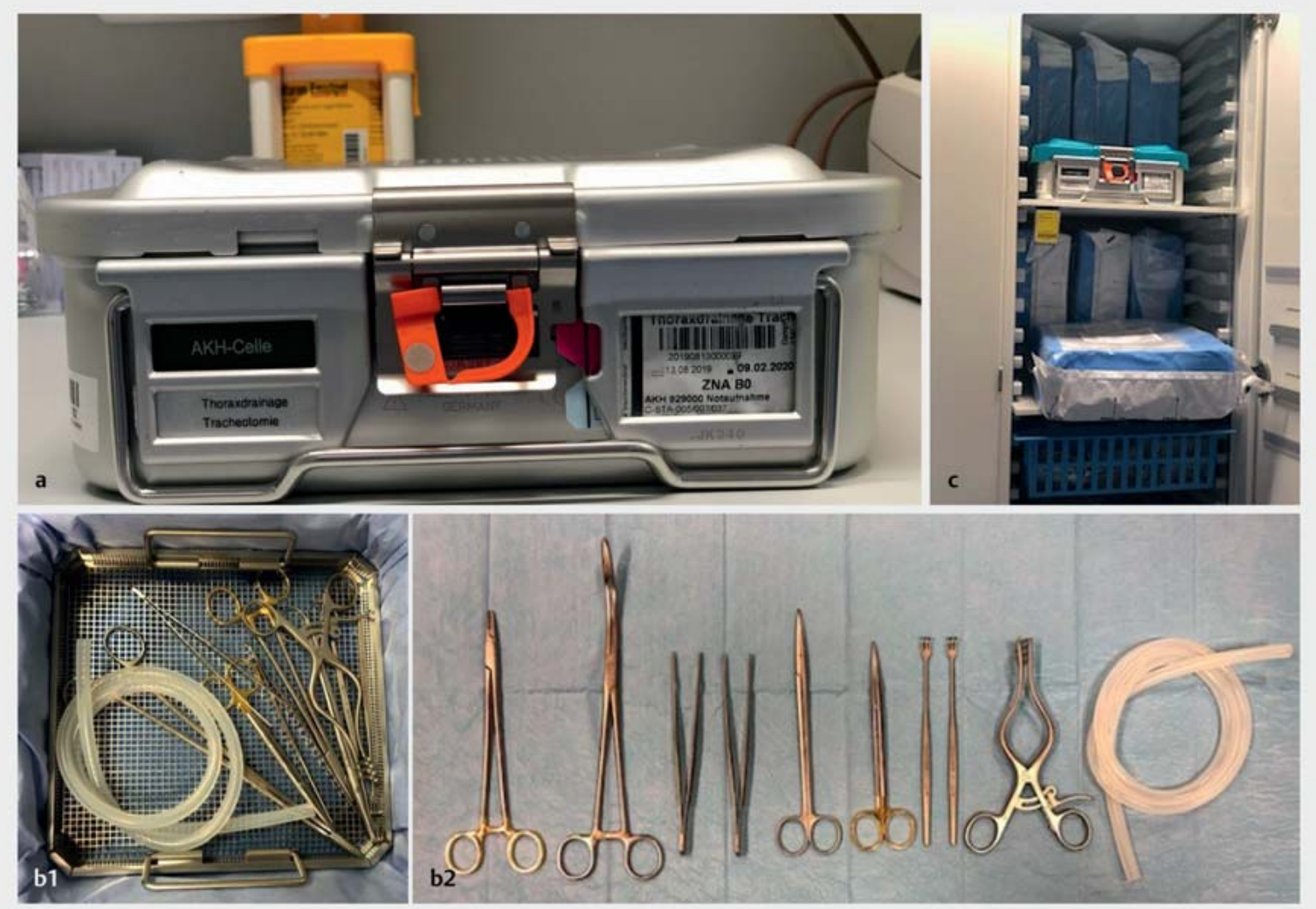

- Abb. 4 a Thoraxdrainagesieb. b Siebinhalt Thoraxdrainagesieb. c Lagerung Thoraxdrainagesieb mit Auffangbehälter im Schrankmodulsystem.

Druckmessung und Volumentherapie, Vorhaltung von Notfall-Blutkonserven (0 negativ) und ein transportabler Monitor mit EKG-Ableitung und ein fahrbarer Behandlungswagen mit notwendigen Notfallmedikamenten zur Kreislauftherapie (z. B. Antiarrhythmika, Katecholamine), sind ebenfalls wichtige Materialien und Geräte im Schockraum.

\section{D - Disability}

Zur Prüfung der Neurologie des polytraumatisierten Patienten sind eine Taschenlampe, eine Nadel/Büroklammer zur Prüfung der Reaktion des Patienten, aber auch ein Blutzuckermessgerät zur Messung des initialen Blutzuckers wichtig.

\section{E - Environment/Exposure}

Zu Environment/Exposure gehören ein Besteck zur vollständigen Entfernung der Kleidung, Lagerungshilfen und vorgewärmte Decken. Für die notwendige erste Wundversorgung sollten entsprechende Nahtmaterialien vorgehalten werden.

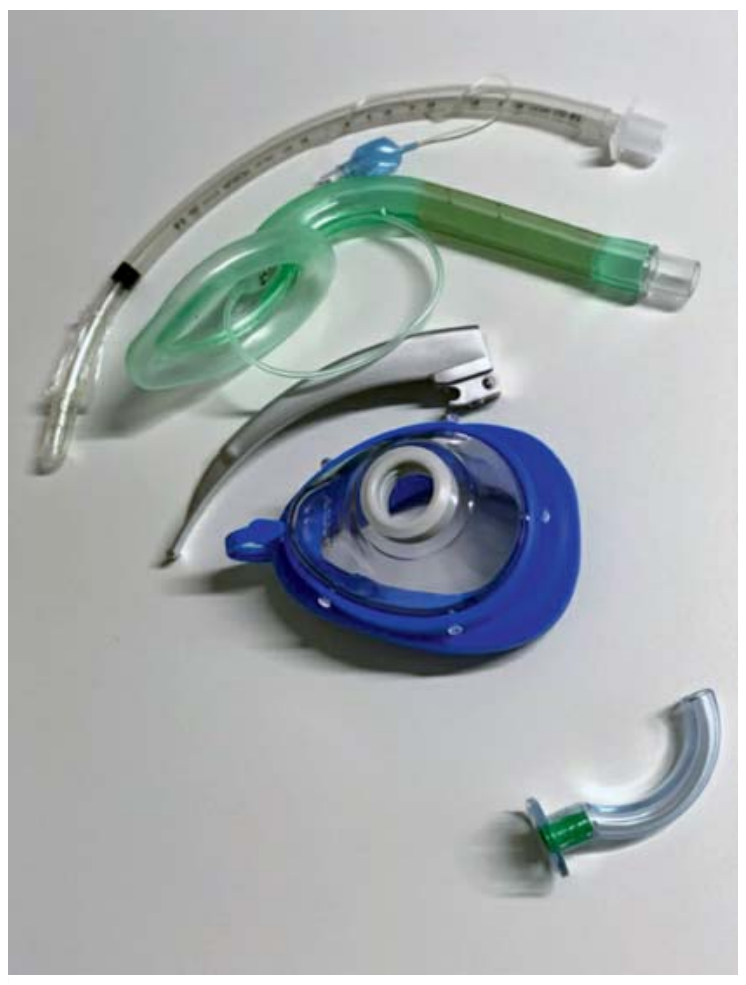

Abb. 5 Intubationsbesteck. 


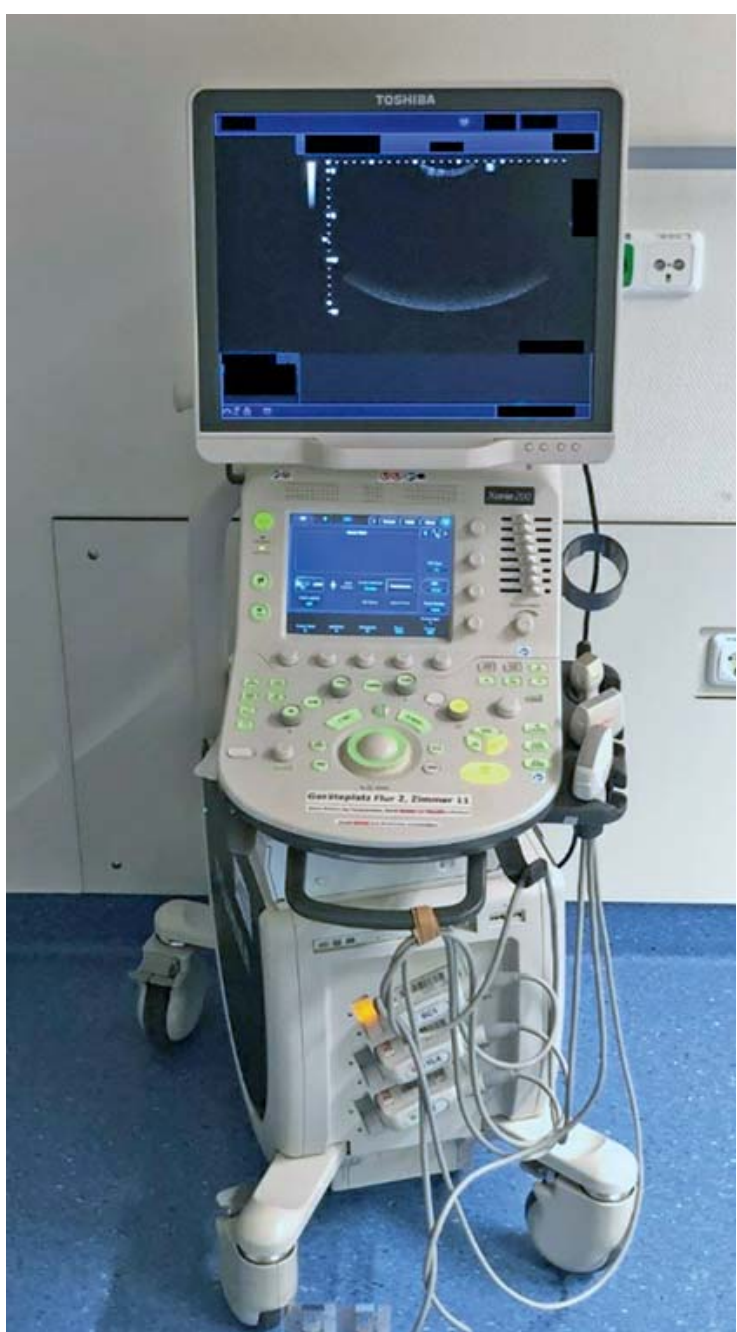

- Abb. 6 Notfall-Sonografiegerät für die eFAST-Sonografie (Fa. Xario 200 Toshiba).

\section{Logistische Materialvorhaltung basie- rend auf dem Schrankmodulsystem}

Das in Schockraum, OP und auf der Intensivstation gleichermaßen verwendete Schrankmodulsystem hat Auswirkungen auf die Materialver- und -entsorgung. Gleichzeitig können der Lagerbestand reduziert und somit auch Vorhaltekosten gespart werden. Durch die reduzierten Lagerbestände können die vorhandenen Lagerkapazitäten besser ausgenutzt werden und dennoch eine ausreichende Versorgung des Schockraums mit Materialien gewährleistet werden.

\section{Wie funktioniert das Schrankmodulsystem?}

Verbrauchslisten der einzelnen Fachabteilungen wurden erarbeitet und den einzelnen Fachabteilungen danach zur Verfügung gestellt. Das Sortiment, welches in das Modulsystem aufgenommen wurde, wurde im Vorfeld mit den Fachabteilungen abgestimmt und festgelegt.
Des Weiteren wurde festgelegt, welche Artikel selten sind und welche Artikel als Durchläufer und Lagerartikel gekennzeichnet werden müssen, da sie häufig verwendet werden. In Zusammenarbeit mit den verantwortlichen Teamleitungen wurden die Sortimente gestrafft und in weiten Teilen standardisiert. Anhand von Bauplänen wurde den Verantwortlichen der Pflegestationen und Fachabteilungen aufgezeigt, an welchem Platz und in welcher Anzahl die Schrankmodule aufgestellt werden. Es wurde im Vorfeld dabei auch bewertet, welche Größe das jeweilige Produkt aufweist und wie viel Platz dafür in einem Korb des Schranks benötigt wird [16].

Die Versorgung und Auffüllung der Materialien des Modulsystems der peripheren Stationen und der ZNA wird durch Versorgungsassistenten der Abteilung Logistik umgesetzt. Die Versorgung des OP- und Anästhesiebereichs erfolgt durch eigene Versorgungsassistenten vor Ort.

Ein Beispiel für die zielgerichtete Lagerung und Versorgung stellt die Standardthoraxdrainage im Schockraum dar. Diese ist als Schrankmodulsystem im Schockraum als Schrankbestandteil festgelegt ( $\mathbf{A b b} \mathbf{4} \mathbf{4}$ c, 7). Die für die Versorgung von mindestens 2 Patienten (Überregionales Traumazentrum) benötigten Mengen an Drainagen sind im Modulsystem im Vorfeld festgelegt worden und es wurden die entsprechenden Reichweiten (Verbrauchszeitraum, Verfall) der Produkte bestimmt. Die festgelegten Mengen der Drainagen, der Aufbewahrungsort und der Zeitraum der Verwendung (Reichweitenbestimmung) werden durch Anlage eines Stammblatts definiert und abgelegt. Die Stammdateneingabe ermöglicht dann die Erstellung von zugehörigen Etiketten mit Barcodes ( $\triangleright$ Abb. 8). Die fertigen Etiketten werden ausgedruckt an die zugehörigen Körbe gesteckt. Bei Verbrauch des vorletzten Artikels dient das Etikett mit Barcode der Nachbestellung und wird durch die verbrauchende Pflegekraft zur Nachbestellung an die Bestellleiste „herausgesteckt“, sodass der Logistikmitarbeiter auf den ersten Blick sieht, was nachbestellt werden muss. Die Anzahl der Nachbestellungen ist definiert und wird letztlich dann über den zentralen Einkauf in der Administration des Krankenhauses ausgelöst. Die Versorgungsassistenten der Logistik scannen morgens ab 06:00 Uhr auf den jeweiligen Stationen die Etiketten an den Nachbestellleisten und räumen die gelieferte Ware (Medical-Produkte und Apothekenartikel) dann wieder in die Schränke ein. Die notwendigen Schulungen der Anwender zur Nutzung des Systems werden in regelmäßigen Abständen wiederholt und somit immer wieder aktualisiert. Ebenso sind die einzelnen Schritte mit definierten Verantwortlichkeiten im Vorfeld festgelegt worden (Pflege: Etikett herausstecken, Logistiker: einscannen und einräumen, Einkauf: Nachbestellung auslösen und Bestands- und Kostenkontrolle). Somit ist ein „Habe-ich-leider-vergessen“-Moment deutlich seltener geworden. 

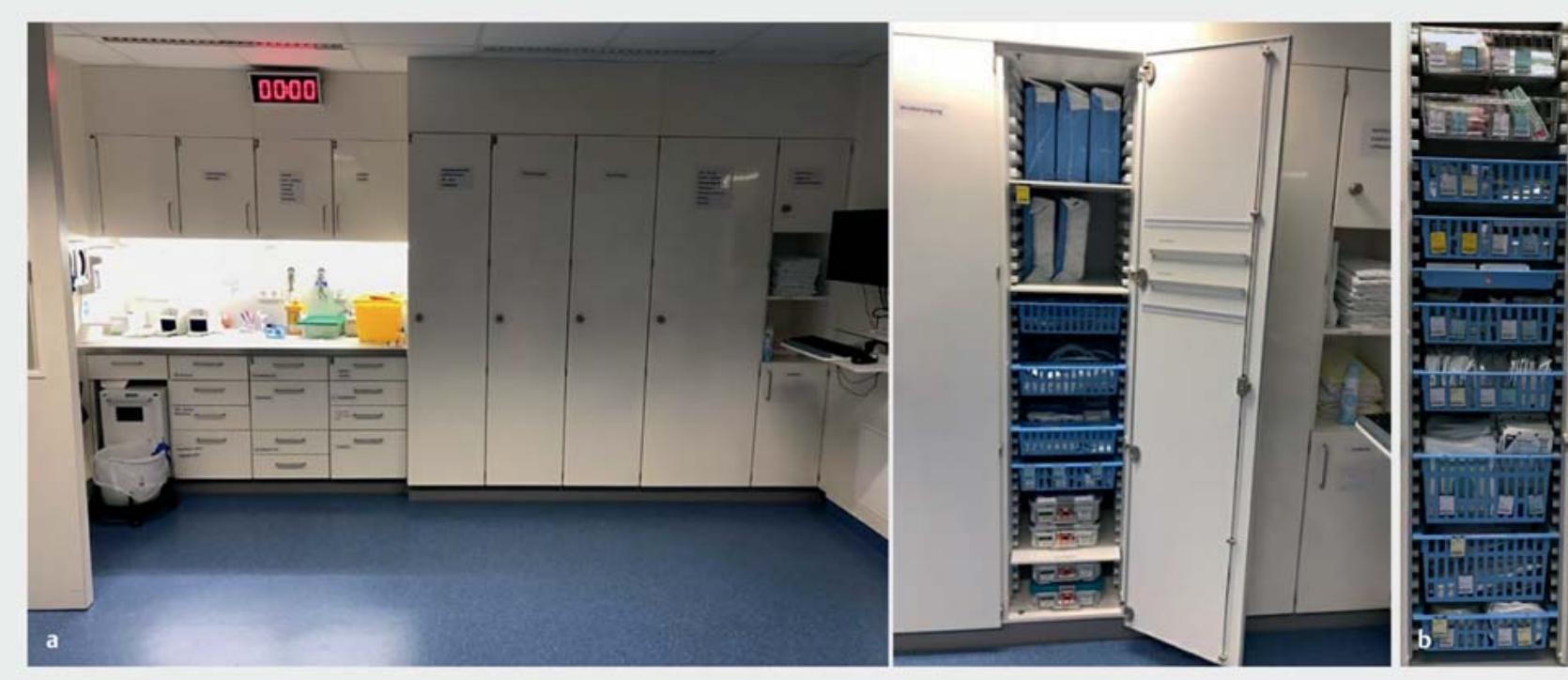

- Abb. 7 a Übersicht des Schrankmodulsystems, rechts geöffnet für die Anlage einer Thoraxdrainage. b Gefülltes Schrankmodulsystem.

\section{FIFO (first in - first out)}

First in - first out, bekannt als FIFO, bezeichnet eine Methode, bei der unterstellt wird, dass zuerst produzierte oder erworbene Produkte auch als Erstes wieder verbraucht werden sollen. Es wird demnach versucht, die ältesten gelagerten Bestände möglichst als Erstes zu verbrauchen, um somit insbesondere einem Produktverlust durch Verfall zu vermeiden. Diese Methode kann sowohl von Individuen als auch von Unternehmen genutzt werden [16].

Dank des Prinzips „first in - first out“ kann die Kapitalbindung durch zu viele Produkte auf den Stationen und in den Fachabteilungen deutlich reduziert werden, da sich die Umschlagshäufigkeit der Produkte erhöht. Ein Verfall der Produkte wird durch Einsatz des Schrankmodulsystems mit FIFO deutlich reduziert und letztlich auch Fehlbestellungen vermieden. Die Stationen und Fachabteilungen wurden vom Aufwand des Auspackens sowie des Verräumens der Materialien durch die eingesetzten Versorgungsassistenten entlastet.

\section{Nach Abschluss der Behandlung im Schockraum}

Nach endgültiger Verlegung des Patienten aus dem Schockraum auf die Intensivstation oder in den OP-Saal werden die Räumlichkeiten (Schockraum und CT-Raum), in denen die Patientenversorgung stattgefunden hat, wieder gereinigt und aufbereitet. Die Ressourcen, die sich in Gebrauch befunden haben, werden gereinigt und desinfiziert. Verbrauchte Materialien werden ersetzt und aufgefüllt. Dieser Vorgang wird durch das Pflegepersonal

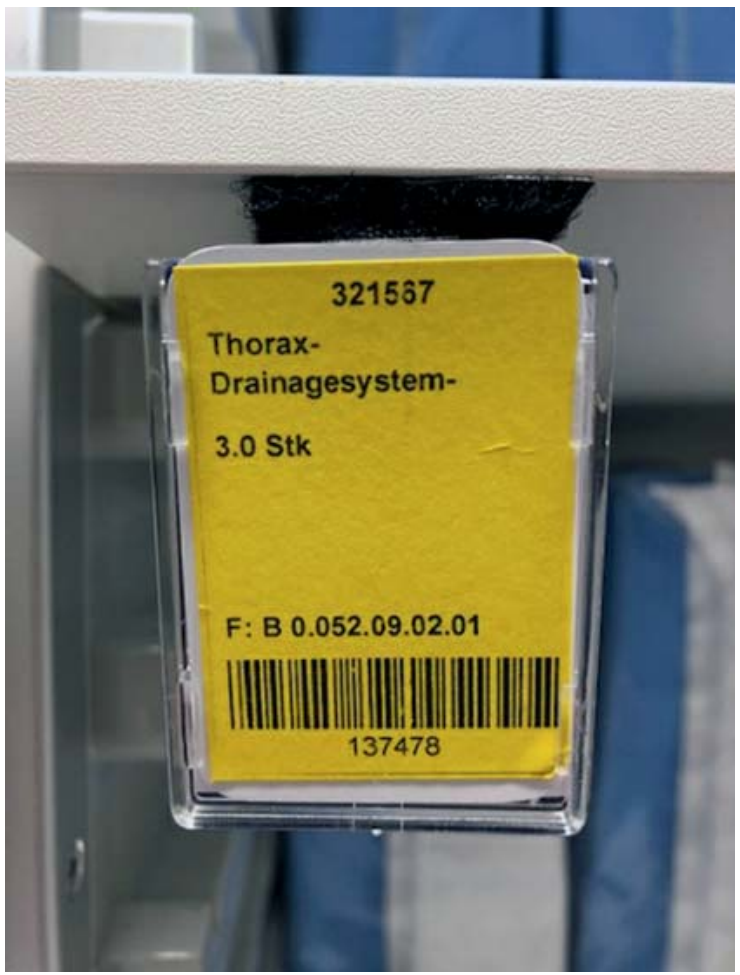

- Abb. 8 Barcodes Thoraxdrainage (festgelegte Mindestmenge 3 Stück).

der ZNA umgehend nach Ende der Patientenversorgung durchgeführt und überprüft.

Die Lagerhaltung und Nachbestellung von Verbrauchsmaterialien und Medikamenten wird durch das Schrankmodulsystem übersichtlich wie oben dargestellt orga- 
nisiert und umgesetzt. Somit sind eine vollständige Materialvorhaltung und ein zeitgerechter Materialverbrauch gewährleistet. Bei Einhaltung der First-in-first-out-Regel werden die Materialien zeitnah verbraucht und das Einhalten der Mindesthaltbarkeitsfristen sichergestellt.

\section{Fazit}

Die Materialvorhaltung und Logistik hat demnach einen ebenso hohen Stellwert in der Gesamtbetrachtung der Polytraumaversorgung wie die direkte Arbeit am schwerverletzten Patienten selbst [16]. Es wird zwar generell davon ausgegangen, dass „alles schon funktioniert“, aber auch die Logistik und Materialwirtschaft sind zu beachtende und zu steuernde Teile der Polytraumaversorgung und sollten daher auch in der Behandlung des schwerverletzten Patienten nicht unterschätzt werden.

\section{Interessenkonflikt}

Die Autorinnen/Autoren geben an, dass kein Interessenkonflikt besteht.

\section{Autorinnen/Autoren}

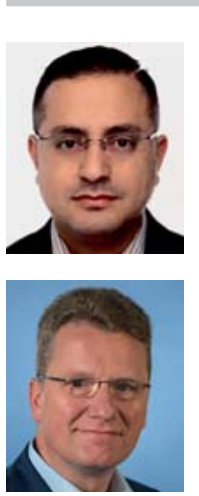

\section{Abd Elgani Haj Yahya}

Facharzt für Unfallchirurgie und Orthopädie. Klinik für Unfallchirurgie, Orthopädie und Neurotraumatologie, Allgemeines Krankenhaus Celle

\section{Ulf Culemann}

Prof. Dr. med., Chefarzt. Klinik für Unfallchirurgie, Orthopädie und Neurotraumatologie, Allgemeines Krankenhaus Celle

\section{Korrespondenzadresse}

\section{Abd Elgani Haj Yahya}

Klinik für Unfallchirurgie, Orthopädie und Neurotraumatologie Allgemeines Krankenhaus Celle

Siemensplatz 4

29223 Celle

Tel.: 05141/721101

Fax: 05141/721109

Abdelgani.HajYahya@akh-celle.de

\section{Literatur}

[1] Lendemans S, Ruchholtz S. S3-Leitlinie Polytrauma/Schwerverletzten-Behandlung. Unfallchirurg 2012; 115: 14-21

[2] Culemann U, Seekamp A, Riedel U et al. Interdisziplinäres Polytraumamanagement. Teil 2: Klinikaufnahme vital bedrohter traumatisierter Patienten. Notfall Rettungsmed 2003; 6: 573-579
[3] Wurmb $\mathrm{T}$, Balling $\mathrm{H}$, Frühwald $\mathrm{P}$ et al. [Polytrauma management in a period of change: time analysis of new strategies for emergency room treatment]. Unfallchirurg 2009; 112: 390-399

[4] American College of Surgeons Committee on Trauma, eds. Resources for optimal Care of the injured Patient. Chicago: American College of Surgeons; 2014

[5] Deutsche Gesellschaft für Unfallchirurgie, Hrsg. DGU-Weißbuch Schwerverletztenversorgung (Vorabdruck). 3. erweiterte Aufl. Berlin: Deutsche Gesellschaft für Unfallchirurgie e.V. (DGU); 2019

[6] Deutsche Gesellschaft für Unfallchirurgie, Hrsg. Weißbuch Schwerverletztenversorgung der DGU. Empfehlungen zur Struktur, Organisation und Ausstattung stationärer Einrichtungen zur Schwerverletzten-Versorgung in der Bundesrepublik Deutschland. Berlin: Deutsche Gesellschaft für Unfallchirurgie e.V.; 2012

[7] Hagemann A, Ideker T. Neuordnung des stationären Heilverfahrens ab 01.01.2013, Rundschreiben der DGUV: Nr. D 14/ 2012, Dokument Nr.411.32/009; 411.36 Anlage 1-3 vom 19.12.2012.

[8] American College of Surgeons; The Committee on Trauma. Advanced Trauma Life Support ${ }^{\circledR}$. Student Course Manual. 10th ed. Chicago: American College of Surgeons; 2018

[9] Bouillon B, Kanz KG, Lackner CK et al. Die Bedeutung des ATLS im Schockraum. Unfallchirurg 2004; 107: 844-850

[10] Thies KC, Nagele P. Advanced Trauma Life Support - Ein Versorgungsstandard für Deutschland? Anaesthesist 2007; 56: 1147-1154

[11] Field JM, Hazinski MF, Sayre M et al. Part 1: executive summary: 2010 American Heart Association Guidelines for Cardiopulmonary Resuscitation and Emergency Cardiovascular Care. Circulation 2010; 122 (18 Suppl. 3): S640-S656

[12] Travers AH, Perkins GD, Berg RA et al. Part 3: Adult Basic Life Support and Automated External Defibrillation: 2015 International Consensus on Cardiopulmonary Resuscitation and Emergency Cardiovascular Care Science with Treatment Recommendations. Circulation 2015; 132 (16 Suppl. 1): S51-S83

[13] Monsieurs KG, Nolan JP, Bossaert LL et al. Kurzdarstellung. Kapitel 1 der Leitlinien zur Reanimation 2015 des European Resuscitation Council. Notfall Rettungsmed 2015; 18: 655747

[14] Barzen S, Schweigkofler U, Hoffmann R. Beckenverletzung, Untersuchung und Anlage des Beckengurts: einfach und praktisch. Notfall Rettungsmed 2019; 22: 154-156

[15] Culemann U, Oestern H, Pohlemann T. Aktuelle Behandlung der Beckenringfraktur. Unfallchirurg 2014; 117: 145-161

[16] Schlepp A, Schick-Leisten M, Jones A et al.; Deutsche Gesellschaft für Sterilgutversorgung e.V. (DGSV). Leitlinie „Lagerung von aufbereiteten Medizinprodukten und Transport zur Verund Entsorgung von aufbereitbaren Medizinprodukten zwischen AEMP und Anwender. Zentralsterilisation 2018; 26 (Suppl.): S1-S48

\section{Bibliografie}

DOI https://doi.org/10.1055/a-1026-9191 Online-publiziert 22.01.2020 | OP-JOURNAL 2020; 36: 41-48 (c) Georg Thieme Verlag KG Stuttgart · New York ISSN 0178-1715 